\title{
Radiative transition probabilities for forbidden lines in singly ionized cobalt (Co II) ${ }^{\star}$
}

\author{
P. Quinet \\ Astrophysique et Spectroscopie, Université de Mons-Hainaut, B-7000 Mons, Belgium
}

Received July 30; accepted September 8, 1997

\begin{abstract}
Radiative transition probabilities have been calculated for the magnetic dipole (M1) and electric quadrupole (E2) transitions connecting the 47 metastable energy levels in the $3 \mathrm{~d}^{8}, 3 \mathrm{~d}^{7} 4 \mathrm{~s}$ and $3 \mathrm{~d}^{6} 4 \mathrm{~s}^{2}$ configurations in Co II. The most important configuration interaction (CI) and relativistic effects have been taken into account in the computations carried out with the help of the relativistic Hartree-Fock (HFR) method combined with a semiempirical optimization of the radial parameters.
\end{abstract}

Key words: atomic data - supernovae: general

\section{Introduction}

In supervova research, the iron group elements such as Fe, $\mathrm{Co}$ and $\mathrm{Ni}$ are of particular interest. Recently, Kuchner et al. (1994) concluded that the cobalt and iron atoms observed through Type Ia supernova (SN Ia) emission lines were produced by the radioactive decay chain ${ }^{56} \mathrm{Ni} \rightarrow{ }^{56} \mathrm{Co}$ $\rightarrow{ }^{56} \mathrm{Fe}$ as predicted by a wide range of previous models for SN Ia explosions (see e.g. Pankey 1962; Colgate \& McKee 1969; Arnett 1979, 1982; Colgate et al. 1980; Weaver et al. 1980; Chevalier 1981; Woosley \& Weaver 1986). According to this model, a small fraction of the decay energy is converted into thermal energy and the expanding shell is cooled mainly through emission in forbidden lines of the first three ionization stages of $\mathrm{Fe}$ and Co produced from excitation of the same nuclei that are daughters of the original ${ }^{56} \mathrm{Ni}$ (see Kirshner \& Oke 1975; Kuchner et al. 1994).

Important atomic processes and reliable atomic data are needed to construct the ionization and thermal structure of Type Ia supernovae at early nebular phase as well as to solve some of the theoretical problems with SN Ia such as the "IR catastrophe" (Fransson et al. 1996). In

\footnotetext{
* Table 3 is also available in electronic form from the CDS via anonymous ftp 130.79.128.5 or http://cdsweb.ustrasbg.fr/Abstract.html
}

this context, it must be noted that, while a large number of transition probabilities have been calculated in the past for the [Fe I], [Fe II], [Fe III], [Ni I], [Ni II] and [Ni III] lines (see e.g. Grevesse et al. 1971; Garstang 1957, 1958, 1962, 1964; Nussbaumer \& Storey 1980, 1982, 1988a; Quinet 1996; Quinet \& Le Dourneuf 1996; Quinet et al. 1996a,b), very few studies of forbidden lines belonging to low ionization stages of cobalt have been reported so far. To our knowledge, only the results obtained by Nussbaumer \& Storey (1988b) for the lines within the ground terms in Co I and Co III and for the forbidden transitions involving the lowest three terms of Co II have been published. This lack of radiative data for forbidden lines of cobalt and, in particular for those belonging to Co II, has been underlined recently by Liu et al. (1997a). In their paper, it is stated that the overall discrepancy between the synthetic and observed spectra of SN Ia is partly caused by the neglect of the [Co II] lines in the spectral synthesis.

The aim of the present work is to provide a new set of accurate transition rates for forbidden lines of singly ionized cobalt. Together, the three low even configurations $3 \mathrm{~d}^{8}, 3 \mathrm{~d}^{7} 4 \mathrm{~s}$ and $3 \mathrm{~d}^{6} 4 \mathrm{~s}^{2}$ in Co II have 47 metastable levels. In our study, radiative transition probabilities for forbidden lines between these metastable levels were calculated using the relativistic Hartree-Fock (HFR) method combined with an optimization of the radial parameters based on the available observed energy levels. The most important configuration interaction (CI) and relativistic effects were included in the calculations. The results reported here can be seen as an extension of the recent investigations of forbidden transitions of astrophysical interest in iron group elements carried out in the cases of Cr II (Quinet 1997), Fe II (Quinet et al. 1996a,b), Fe III (Quinet 1996), Ni I and Ni II (Quinet \& Le Dourneuf 1996).

\section{Method used}

For the calculations, we used the suite of computer programs due to Cowan (1981) within the framework of the approximately relativistic Hartree-Fock (HFR) method originally introduced by Cowan \& Griffin (1976). 
Considerable amount of CI effects were included explicitly in the calculations. These configurations are $3 \mathrm{~d}^{8}, 3 \mathrm{~d}^{7} 4 \mathrm{~s}$, $3 \mathrm{~d}^{7} 5 \mathrm{~s}, 3 \mathrm{~d}^{7} 4 \mathrm{~d}, 3 \mathrm{~d}^{7} 5 \mathrm{~d}, 3 \mathrm{~d}^{6} 4 \mathrm{~s}^{2}, 3 \mathrm{~d}^{6} 4 \mathrm{p}^{2}, 3 \mathrm{~d}^{6} 4 \mathrm{~d}^{2}$ and $3 \mathrm{~d}^{6} 4 \mathrm{~s} 4 \mathrm{~d}$. In order to reduce as much as possible the discrepancies between computed and observed energy levels, the HFR technique was used in combination with a well known least-squares optimization of the radial parameters.

The fitting procedure was applied to the $3 \mathrm{~d}^{8}$ and $3 \mathrm{~d}^{7} 4 \mathrm{~s}$ configurations of Co II with the experimental energy levels compiled by Sugar \& Corliss (1985). In the absence of CI, the $3 \mathrm{~d}^{8}$ configuration is described by four parameters, $E_{\mathrm{av}}, F^{2}(3 \mathrm{~d}, 3 \mathrm{~d}), F^{4}(3 \mathrm{~d}, 3 \mathrm{~d})$ and $\zeta_{3 \mathrm{~d}}$, while for the the $3 \mathrm{~d}^{7} 4 \mathrm{~s}$, also the $G^{2}(3 \mathrm{~d}, 4 \mathrm{~s})$ parameter is required. In addition to the explicit introduction of CI mentioned above, effective interaction parameters such as $\alpha$ and $\beta$ (see Trees 1951a,b; Racah 1952), associated with the excitation out of the $3 \mathrm{~s}$ and $3 \mathrm{p}$ subshells into the $3 \mathrm{~d}$, can be used to describe the cumulative effects of distant configurations on $3 \mathrm{~d}^{8}$ and $3 \mathrm{~d}^{7} 4 \mathrm{~s}$. All the parameters of these two configurations were adjusted except the effective interaction parameter $\beta$ corresponding to $3 \mathrm{~d}^{8}$. Indeed, for this configuration, only four electrostatic parameters $\left(E_{\mathrm{av}}, F^{2}(3 \mathrm{~d}\right.$, $3 \mathrm{~d}), F^{4}(3 \mathrm{~d}, 3 \mathrm{~d})$ and $\left.\alpha\right)$ can be optimized due to the fact that only the four terms ${ }^{3} \mathrm{~F},{ }^{3} \mathrm{P},{ }^{1} \mathrm{D}$ and ${ }^{1} \mathrm{G}$ have been determined experimentally (the ${ }^{1} \mathrm{~S}$ is missing). The average energy of the $3 \mathrm{~d}^{6} 4 \mathrm{~s}^{2}$ configuration was also adjusted using the $\mathrm{a}^{5} \mathrm{D}$ multiplet. The $\mathrm{F}^{k}, \mathrm{G}^{k}$ and $\mathrm{R}^{k}$ integrals not optimized were arbitrarily scaled down by a factor 0.85 while ab initio values of the spin-orbit integrals, $\zeta$, computed by the Blume-Watson method, were used without scaling.

The parameter values adopted for the $3 \mathrm{~d}^{8}, 3 \mathrm{~d}^{7} 4 \mathrm{~s}$ and $3 \mathrm{~d}^{6} 4 \mathrm{~s}^{2}$ configurations of Co II are reported in Table 1 while calculated energy levels and Landé $g$-factors are compared with the experimental values in Table 2 . In agreement with previous classifications, terms having the same LS notation are distinguished by the letters $\mathrm{a}, \mathrm{b}$ and $c$ in increasing energy order. As seen from Table 2, our calculated energy levels differ from the experimental data by amounts typically lower than $1 \%$, the largest difference $\left(289 \mathrm{~cm}^{-1}\right)$ being observed for $3 \mathrm{~d}^{7}\left({ }^{2} \mathrm{G}\right) 4 \mathrm{~s} \mathrm{a}^{3} \mathrm{G}_{3}$ at $22415 \mathrm{~cm}^{-1}$. A very nice agreement is also observed when comparing the Landé $g$-factors obtained in our work with the measured values (when available).

\section{Transition probabilities}

The transition probabilities, $A_{k i}$, were calculated for magnetic dipole (M1) and electric quadrupole (E2) lines connecting the 47 energetically lowest levels belonging to the $3 \mathrm{~d}^{8}, 3 \mathrm{~d}^{7} 4 \mathrm{~s}$ and $3 \mathrm{~d}^{6} 4 \mathrm{~s}^{2}$ configurations of Co II, i.e. the transitions in which both energy levels are below the lowest level of odd parity.

Table 3 contains the experimental wavelengths deduced from the observed energy levels (Sugar \& Corliss $1985)$ and the $A$-values calculated in the present work. All the calculations were carried out using the computed
Table 1. Values of the parameters $\left(\right.$ in $\mathrm{cm}^{-1}$ ) adopted in the HFR calculations for the $3 \mathrm{~d}^{8}, 3 \mathrm{~d}^{7} 4 \mathrm{~s}$ and $3 \mathrm{~d}^{6} 4 \mathrm{~s}^{2}$ configurations of Co II. The ratios between fitted and ab initio HFR values are also indicated

\begin{tabular}{|c|c|c|c|}
\hline \multicolumn{4}{|c|}{ Configuration Parameter Fitted value Ratio ${ }^{b}$} \\
\hline \multirow[t]{4}{*}{$3 d^{8}$} & $\begin{array}{c}E_{\mathrm{av}} \\
F^{2}(3 \mathrm{~d}, 3 \mathrm{~d})\end{array}$ & $\begin{array}{l}12516 . \\
71848 .\end{array}$ & 0.8541 \\
\hline & $F^{4}(3 \mathrm{~d}, 3 \mathrm{~d})$ & 45288. & 0.8731 \\
\hline & $\alpha$ & 79. & \\
\hline & $\zeta_{3 \mathrm{~d}}$ & 469. & 0.9915 \\
\hline \multirow[t]{7}{*}{$3 d^{7} 4 s$} & $E_{\mathrm{av}}$ & 27557. & \\
\hline & $F^{2}(3 \mathrm{~d}, 3 \mathrm{~d})$ & 78543. & 0.8527 \\
\hline & $F^{4}(3 \mathrm{~d}, 3 \mathrm{~d})$ & 51650. & 0.9038 \\
\hline & $\alpha$ & 91. & \\
\hline & $\beta$ & -1035. & \\
\hline & $G^{2}(3 \mathrm{~d}, 4 \mathrm{~s})$ & 8467. & 0.8652 \\
\hline & $\zeta_{3 \mathrm{~d}}$ & 507. & 0.9657 \\
\hline \multirow[t]{4}{*}{$3 d^{6} 4 s^{2}$} & $E_{\mathrm{av}}$ & 78765. & \\
\hline & $F^{2}(3 \mathrm{~d}, 3 \mathrm{~d})$ & $84612 .^{a}$ & 0.8500 \\
\hline & $F^{4}(3 \mathrm{~d}, 3 \mathrm{~d})$ & $52787 .^{a}$ & 0.8500 \\
\hline & $\zeta_{3 \mathrm{~d}}$ & $581 .^{a}$ & 1.0000 \\
\hline
\end{tabular}

${ }^{a}$ Fixed to the ab initio value.

${ }^{b}$ Ratio $=$ Fitted $/$ ab initio.

energy intervals but the use of experimental energy differences would only produce a marginal change in the final results. If the two types of radiation (M1 and E2) contribute significantly to the total intensity of a line, the sum of both components is given. The exclusion criterion of one particular type of radiation for a given transition is that the corresponding $A$-value should be less than $1 \%$ of the sum of M1 and E2 contributions. Owing to the extensive nature of the results, only transitions for which $A_{k i}$ is greater than $0.001 \mathrm{~s}^{-1}$ are reported in the table. The complete data can be obtained upon request to the author.

As already noticed for other ions, examining the results, it is seen that for most of the strong multiplets either magnetic dipole radiation predominates (electric quadrupole radiation being negligible) or electric quadrupole radiation predominates (magnetic dipole radiation being negligible). In the former case, the transitions are mostly intercombination multiplets $(\Delta S \neq 0)$ made possible by spin-orbit interaction. In the latter case, the transitions are mostly allowed by the $L S$-coupling selection rules for electric quadrupole radiation (i.e. $\Delta S=0$, $\Delta L=0, \pm 1, \pm 2)$. There are few transitions in which the two types of radiation are of comparable intensities.

The calculation of line strengths and transition probabilities involves the evaluation of the sum of several terms resulting of intermediate coupling and/or configuration interaction mixing of basis states. When strong basis function mixing is present, there frequently are destructive 
Table 2. Calculated HFR energy levels (in $\mathrm{cm}^{-1}$ ) and Landé $g$-factors and comparison with experiment for the $3 \mathrm{~d}^{8}, 3 \mathrm{~d}^{7} 4 \mathrm{~s}$ and $3 \mathrm{~d}^{6} 4 \mathrm{~s}^{2}$ configurations of Co II

\begin{tabular}{|c|c|c|c|c|c|}
\hline Configuration & Term & & $E_{\mathrm{obs}}{ }^{a} g_{\mathrm{obs}}{ }^{a}$ & $E_{\text {calc }} g_{\text {calc }}$ & $\Delta E^{b}$ \\
\hline \multirow[t]{3}{*}{$3 \mathrm{~d}^{8}$} & $a^{3} F$ & 4 & 0. & 0.1 .250 & 0 . \\
\hline & & 3 & 951 & 948.1 .084 & 3 . \\
\hline & & 2 & 1597. & 1598. 0.668 & -1 \\
\hline \multirow[t]{5}{*}{$3 \mathrm{~d}^{7}\left({ }^{4} \mathrm{~F}\right) 4 \mathrm{~s}$} & $a^{5} \mathrm{~F}$ & 5 & 3351.1 .413 & 3397. 1.401 & -46 . \\
\hline & & 4 & 4029. 1.354 & 4035. 1.350 & -6 . \\
\hline & & 3 & 4561.1 .258 & 4539. 1.250 & 22 . \\
\hline & & 2 & 4950. 0.997 & 4911. 1.000 & 39. \\
\hline & & 1 & 5205.0 .00 & 5155.0 .000 & 50. \\
\hline \multirow[t]{3}{*}{$3 d^{7}\left({ }^{4} F\right) 4 s$} & $b^{3} \mathrm{~F}$ & 4 & 9813. 1.243 & 9820.1 .250 & -7 . \\
\hline & & & 10708. 1.082 & 10681. 1.084 & 27 . \\
\hline & & & 11322.0.68 & 11272.0 .679 & 50. \\
\hline $3 d^{8}$ & $a^{1} D$ & & 11651. 1.111 & 11648. 1.096 & 3. \\
\hline \multirow[t]{3}{*}{$3 d^{8}$} & $a^{3} \mathrm{P}$ & & 13261. 1.415 & 13275. 1.392 & -14 \\
\hline & & & 13404. 1.484 & 13390. 1.502 & 14. \\
\hline & & & 13593. & 13584 . & 9. \\
\hline \multirow{3}{*}{$3 d^{7}\left({ }^{4} \mathrm{P}\right) 4 \mathrm{~s}$} & $a^{5} \mathrm{P}$ & & 17772.1 .68 & 17786. 1.668 & -14 \\
\hline & & & 18032.1 .839 & 18064.1 .829 & -32 . \\
\hline & & & 18339. 2.510 & 18372. 2.493 & -33 . \\
\hline $3 d^{8}$ & $a^{1} G$ & & 19190. & 19193. 1.000 & -3 . \\
\hline \multirow[t]{3}{*}{$3 \mathrm{~d}^{7}\left({ }^{2} \mathrm{G}\right) 4 \mathrm{~s}$} & $a^{3} G$ & 52 & 21625. 1.186 & 21390. 1.199 & 235 . \\
\hline & & & 22009. 1.06 & 21754.1 .048 & 255. \\
\hline & & & 22415. 0.75 & 22126. 0.750 & 289. \\
\hline \multirow[t]{3}{*}{$3 d^{7}\left({ }^{4} \mathrm{P}\right) 4 \mathrm{~s}$} & $b^{3} \mathrm{P}$ & & 24075. 1.500 & 23998. 1.497 & 77 . \\
\hline & & & 24268. 1.498 & 24260.1 .471 & 8. \\
\hline & & & 24411. & 24468 & -57 \\
\hline \multirow[t]{3}{*}{$3 \mathrm{~d}^{7}\left({ }^{2} \mathrm{P}\right) 4 \mathrm{~s}$} & $c^{3} \mathrm{P}$ & & 24887. 1.49 & 25149. 1.482 & -262 . \\
\hline & & & 25318. 1.47 & 25525.1 .451 & -207 . \\
\hline & & & 25862. & 26004. & -142 . \\
\hline $3 d^{7}\left({ }^{2} G\right) 4 s$ & $b^{1} G$ & 42 & 25147. 0.997 & 25100. 0.994 & 47. \\
\hline \multirow[t]{3}{*}{$3 \mathrm{~d}^{7}\left({ }^{2} \mathrm{H}\right) 4 \mathrm{~s}$} & $a^{3} \mathrm{H}$ & 62 & 27106. 1.172 & 27316.1 .167 & -210 . \\
\hline & & & 27469. 1.041 & 27644.1 .034 & -175 . \\
\hline & & & 27902. 0.803 & 28037.0 .809 & -135 . \\
\hline \multirow[t]{3}{*}{$3 \mathrm{~d}^{7}\left({ }^{2} \mathrm{D}\right) 4 \mathrm{~s}$} & $a^{3} D$ & & 27485. 1.36 & 27495. 1.334 & -10 . \\
\hline & & & 28112. 1.18 & 28071. 1.182 & 41. \\
\hline & & & 29269. 0.77 & 29229. 0.791 & 40. \\
\hline $3 \mathrm{~d}^{7}\left({ }^{2} \mathrm{P}\right) 4 \mathrm{~s}$ & $a^{1} \mathrm{P}$ & & 27585. 0.83 & 27626. 0.798 & -41 . \\
\hline $3 \mathrm{~d}^{7}\left({ }^{2} \mathrm{H}\right) 4 \mathrm{~s}$ & $a^{1} \mathrm{H}$ & & 30567. 1.027 & 30782.1 .001 & -215 . \\
\hline $3 \mathrm{~d}^{7}\left({ }^{2} \mathrm{D}\right) 4 \mathrm{~s}$ & $\mathrm{~b}^{1} \mathrm{D}$ & & 31199. 1.02 & 31196. 1.012 & 3. \\
\hline \multirow[t]{5}{*}{$3 d^{6} 4 s^{2}$} & $a^{5} \mathrm{D}$ & & 40696. & 40707. 1.499 & -11 \\
\hline & & & 41314. & 41314. 1.501 & 0 . \\
\hline & & & 41738. & 41734. 1.501 & 4. \\
\hline & & & 42009. & 42004. 1.501 & 5 . \\
\hline & & 0 & & 42136. & \\
\hline \multirow[t]{3}{*}{$3 \mathrm{~d}^{7}\left({ }^{2} \mathrm{~F}\right) 4 \mathrm{~s}$} & $c^{3} \mathrm{~F}$ & & 40771. & 40649. 0.666 & 122. \\
\hline & & & 40879. & 40780. 1.084 & 99. \\
\hline & & & 41047. & 40982.1 .252 & 105. \\
\hline $3 d^{7}\left({ }^{2} F\right) 4 s$ & $a^{1} \mathrm{~F}$ & 34 & 44091. & 43974. 1.000 & 117. \\
\hline
\end{tabular}

${ }^{a}$ Sugar \& Corliss (1985).

${ }^{b} \Delta E=E_{\text {obs }}-E_{\text {calc }}$.
Table 3. Radiative transition probabilities, $A_{k i}$ in s ${ }^{-1}$, as calculated in the present work for forbidden lines of Co II. $A(B)$ stands for $A \cdot 10^{B}$. Only transitions for which $A_{k i}$ is greater than $0.001 \mathrm{~s}^{-1}$ are reported

\begin{tabular}{|c|c|c|c|}
\hline Multiplet & $J-J^{\prime}$ & $\lambda(\AA)^{a}$ Type & $A_{k i}$ \\
\hline \multirow[t]{2}{*}{$a^{3} F-a^{3} F$} & $4-3$ & $105178.00 \mathrm{M} 1$ & $2.21(-2)$ \\
\hline & $3-2$ & $154562.77 \mathrm{M} 1$ & $9.88(-3)$ \\
\hline \multirow[t]{8}{*}{$a^{3} F-b^{3} F$} & $4-4$ & 10187.81 E2 & $5.36(-2)$ \\
\hline & $4-3$ & $9335.84 \mathrm{E} 2$ & $2.30(-2)$ \\
\hline & $4-2$ & 8829.97 E2 & $1.01(-3)$ \\
\hline & $3-4$ & $11280.47 \mathrm{E} 2$ & $6.33(-3)$ \\
\hline & $3-3$ & $10245.24 \mathrm{E} 2$ & $3.47(-2)$ \\
\hline & $3-2$ & 9639.21 M1,E2 & $3.16(-2)$ \\
\hline & $2-3$ & $10972.56 \mathrm{E} 2$ & $8.74(-3)$ \\
\hline & $2-2$ & 10280.34 M1,E2 & $4.06(-2)$ \\
\hline \multirow[t]{3}{*}{$a^{3} F-a^{1} D$} & $4-2$ & $8580.24 \mathrm{E} 2$ & $9.74(-3)$ \\
\hline & $3-2$ & $9342.38 \mathrm{M} 1$ & $1.74(-1)$ \\
\hline & $2-2$ & $9943.41 \mathrm{M} 1, \mathrm{E} 2$ & $8.16(-2)$ \\
\hline \multirow[t]{6}{*}{$a^{3} F-a^{3} P$} & $4-2$ & $7538.96 \mathrm{E} 2$ & $4.98(-2)$ \\
\hline & $3-2$ & 8121.07 M1,E2 & $6.70(-2)$ \\
\hline & $3-1$ & $8027.36 \mathrm{E} 2$ & $4.20(-2)$ \\
\hline & $2-2$ & 8571.44 M1,E2 & $1.63(-2)$ \\
\hline & $2-1$ & 8467.11 M1,E2 & $1.68(-2)$ \\
\hline & $2-0$ & $8333.82 \mathrm{E} 2$ & $5.34(-2)$ \\
\hline \multirow[t]{2}{*}{$a^{3} F-a^{5} P$} & $4-2$ & $5544.26 \mathrm{E} 2$ & $3.42(-3)$ \\
\hline & $3-1$ & $5749.40 \mathrm{E} 2$ & $1.13(-3)$ \\
\hline \multirow[t]{2}{*}{$a^{3} F-a^{1} G$} & $4-4$ & $5209.57 \mathrm{M} 1$ & $1.36(-1)$ \\
\hline & $3-4$ & $5481.05 \mathrm{M} 1, \mathrm{E} 2$ & $7.03(-2)$ \\
\hline \multirow[t]{8}{*}{$a^{3} F-a^{3} G$} & $4-5$ & $4623.06 \mathrm{E} 2$ & $7.73(-1)$ \\
\hline & $4-4$ & $4542.23 \mathrm{E} 2$ & $1.22(-1)$ \\
\hline & $4-3$ & $4460.13 \mathrm{E} 2$ & $6.87(-3)$ \\
\hline & $3-5$ & $4835.61 \mathrm{E} 2$ & $3.33(-2)$ \\
\hline & $3-4$ & $4747.24 \mathrm{E} 2$ & $5.61(-1)$ \\
\hline & $3-3$ & $4657.64 \mathrm{E} 2$ & $1.49(-1)$ \\
\hline & $2-4$ & $4897.68 \mathrm{E} 2$ & $3.76(-2)$ \\
\hline & $2-3$ & $4802.36 \mathrm{E} 2$ & $5.31(-1)$ \\
\hline \multirow[t]{6}{*}{$a^{3} F-b^{3} P$} & $4-2$ & $4152.58 \mathrm{E} 2$ & $3.12(+0)$ \\
\hline & $3-2$ & $4323.28 \mathrm{E} 2$ & $5.12(-1)$ \\
\hline & $3-1$ & $4287.50 \mathrm{E} 2$ & $9.37(-1)$ \\
\hline & $2-2$ & $4447.69 \mathrm{E} 2$ & $4.72(-2)$ \\
\hline & $2-1$ & $4409.83 \mathrm{E} 2$ & $5.47(-1)$ \\
\hline & $2-0$ & $4382.03 \mathrm{E} 2$ & $8.77(-1)$ \\
\hline \multirow[t]{5}{*}{$a^{3} F-c^{3} P$} & $4-2$ & 4017.10 E2 & $2.66(-1)$ \\
\hline & $3-1$ & $4102.73 \mathrm{E} 2$ & $2.03(+0)$ \\
\hline & $2-2$ & $4292.62 \mathrm{E} 2$ & $4.32(-3)$ \\
\hline & $2-1$ & $4214.61 \mathrm{E} 2$ & $3.97(-1)$ \\
\hline & $2-0$ & $4120.12 \mathrm{E} 2$ & $3.10(+0)$ \\
\hline \multirow[t]{3}{*}{$a^{3} F-b^{1} G$} & $4-4$ & 3975.43 M1,E2 & $2.07(-2)$ \\
\hline & $3-4$ & $4131.60 \mathrm{M} 1, \mathrm{E} 2$ & $4.99(-3)^{*}$ \\
\hline & $2-4$ & $4245.08 \mathrm{E} 2$ & $1.04(-1)$ \\
\hline \multirow[t]{6}{*}{$\mathrm{a}^{3} \mathrm{~F}-\mathrm{a}^{3} \mathrm{H}$} & $4-6$ & $3688.18 \mathrm{E} 2$ & $2.66(+0)$ \\
\hline & $4-5$ & $3639.41 \mathrm{E} 2$ & $1.42(-1)$ \\
\hline & $4-4$ & $3582.90 \mathrm{M} 1, \mathrm{E} 2$ & $6.10(-3)$ \\
\hline & $3-5$ & $3769.86 \mathrm{E} 2$ & $2.23(+0)$ \\
\hline & $3-4$ & $3709.26 \mathrm{E} 2$ & $1.86(-1)$ \\
\hline & $2-4$ & $3800.47 \mathrm{E} 2$ & $1.93(+0)$ \\
\hline
\end{tabular}


Table 3. continued

\begin{tabular}{|c|c|c|c|}
\hline \multicolumn{2}{|c|}{ Multiplet $J-J^{\prime}$} & \multirow{2}{*}{$\frac{\lambda(\AA)^{a} \text { Type }}{3637.36 \text { E2 }}$} & \multirow{2}{*}{$\frac{A_{k i}}{1.59(+0)}$} \\
\hline$a^{3} F-a^{3} D$ & $4-3$ & & \\
\hline & $4-2$ & $3556.18 \mathrm{E} 2$ & $8.62(-2)$ \\
\hline & $3-3$ & $3767.66 \mathrm{E} 2$ & $4.60(-1)$ \\
\hline & $3-2$ & $3680.63 \mathrm{E} 2$ & $1.26(+0)$ \\
\hline & $3-1$ & $3530.26 \mathrm{E} 2$ & $2.88(-2)$ \\
\hline & $2-3$ & $3861.80 \mathrm{E} 2$ & $4.54(-2)$ \\
\hline & $2-2$ & $3770.42 \mathrm{E} 2$ & $6.30(-1)$ \\
\hline & $2-1$ & $3612.78 \mathrm{E} 2$ & $8.77(-1)$ \\
\hline \multirow[t]{2}{*}{$a^{3} \mathrm{~F}-\mathrm{a}^{1} \mathrm{P}$} & $3-1$ & $3753.42 \mathrm{E} 2$ & $2.64(-1)$ \\
\hline & $2-1$ & $3846.84 \mathrm{E} 2$ & $7.53(-1)$ \\
\hline$a^{3} \mathrm{~F}-\mathrm{a}^{1} \mathrm{H}$ & $3-5$ & $3375.49 \mathrm{E} 2$ & $5.52(-2)$ \\
\hline \multirow[t]{2}{*}{$a^{3} F-b^{1} D$} & $3-2$ & $3304.94 \mathrm{M} 1, \mathrm{E} 2$ & $1.60(-2)$ \\
\hline & $2-2$ & 3377.16 M1,E2 & $5.60(-3)^{*}$ \\
\hline \multirow[t]{4}{*}{$a^{3} F-a^{5} D$} & $4-4$ & $2456.52 \mathrm{M} 1, \mathrm{E} 2$ & $2.53(-2)^{*}$ \\
\hline & $4-3$ & 2419.74 M1,E2 & $1.76(-3)$ \\
\hline & $3-4$ & 2515.28 M1,E2 & $5.70(-3)$ \\
\hline & $3-3$ & 2476.73 M1,E2 & $1.83(-3)$ \\
\hline \multirow[t]{9}{*}{$a^{3} F-c^{3} F$} & $4-2$ & $2451.97 \mathrm{E} 2$ & $5.88(-2)$ \\
\hline & $4-3$ & $2445.48 \mathrm{E} 2$ & $8.87(-1)$ \\
\hline & $4-4$ & $2435.49 \mathrm{E} 2$ & $2.92(+0)$ \\
\hline & $3-2$ & $2510.51 \mathrm{E} 2$ & $1.08(+0)$ \\
\hline & $3-3$ & $2503.70 \mathrm{E} 2$ & $1.80(+0)$ \\
\hline & $3-4$ & $2493.23 \mathrm{E} 2$ & $6.84(-1)$ \\
\hline & $2-2$ & $2551.96 \mathrm{E} 2$ & $2.18(+0)$ \\
\hline & $2-3$ & $2544.92 \mathrm{E} 2$ & $8.00(-1)$ \\
\hline & $2-4$ & $2534.11 \mathrm{E} 2$ & $3.04(-2)$ \\
\hline \multirow[t]{3}{*}{$a^{3} F-a^{1} F$} & $4-3$ & $2267.34 \mathrm{E} 2$ & $1.83(-2)$ \\
\hline & $3-3$ & $2317.30 \mathrm{E} 2$ & $1.59(-2)$ \\
\hline & $2-3$ & $2352.57 \mathrm{E} 2$ & $2.14(-2)$ \\
\hline \multirow[t]{4}{*}{$a^{5} F-a^{5} F$} & $5-4$ & 147361.14 M1 & $1.03(-2)$ \\
\hline & $4-3$ & 187985.83 M1 & $9.27(-3)$ \\
\hline & $3-2$ & 256741.94 M1 & $5.00(-3)$ \\
\hline & $2-1$ & 392635.09 M1 & $1.57(-3)$ \\
\hline \multirow[t]{8}{*}{$a^{5} \mathrm{~F}-\mathrm{b}^{3} \mathrm{~F}$} & $5-4$ & $15469.95 \mathrm{M} 1$ & $2.66(-2)$ \\
\hline & $4-4$ & $17284.47 \mathrm{M} 1$ & $2.98(-3)^{*}$ \\
\hline & $4-3$ & 14967.16 M1 & $4.17(-3)$ \\
\hline & $3-4$ & 19034.62 M1 & $4.81(-3)$ \\
\hline & $3-3$ & 16261.91 M1 & $4.53(-3)^{*}$ \\
\hline & $2-3$ & $17361.59 \mathrm{M} 1$ & $9.59(-3)$ \\
\hline & $2-2$ & 15689.96 M1 & $4.34(-3)$ \\
\hline & $1-2$ & $16343.04 \mathrm{M} 1$ & $1.34(-2)$ \\
\hline \multirow[t]{10}{*}{$a^{5} F-a^{5} P$} & $5-3$ & $6932.36 \mathrm{E} 2$ & $3.74(-2)$ \\
\hline & $4-3$ & $7274.58 \mathrm{M} 1, \mathrm{E} 2$ & $1.14(-2)$ \\
\hline & $4-2$ & $7139.54 \mathrm{E} 2$ & $2.10(-2)$ \\
\hline & $3-3$ & $7567.42 \mathrm{M} 1, \mathrm{E} 2$ & $2.61(-3)$ \\
\hline & $3-2$ & $7421.40 \mathrm{E} 2$ & $1.46(-2)$ \\
\hline & $3-1$ & $7255.95 \mathrm{E} 2$ & $1.10(-2)$ \\
\hline & $2-2$ & $7642.31 \mathrm{E} 2$ & $6.10(-3)$ \\
\hline & $2-1$ & $7466.98 \mathrm{E} 2$ & $1.59(-2)$ \\
\hline & $1-2$ & $7794.01 \mathrm{M} 1, \mathrm{E} 2$ & $1.30(-3)$ \\
\hline & $1-1$ & $7611.74 \mathrm{E} 2$ & $1.30(-2)$ \\
\hline \multirow[t]{8}{*}{$a^{5} F-a^{3} G$} & $5-5$ & $5470.72 \mathrm{M} 1$ & $3.73(-1)^{*}$ \\
\hline & $5-4$ & $5357.89 \mathrm{M} 1$ & $2.17(-2)$ \\
\hline & $4-5$ & 5681.65 M1 & $7.54(-2)$ \\
\hline & $4-4$ & $5560.05 \mathrm{M} 1$ & $2.11(-1)$ \\
\hline & $4-3$ & $5437.53 \mathrm{M} 1$ & $1.37(-2)$ \\
\hline & $3-4$ & $5729.51 \mathrm{M} 1$ & $9.24(-2)$ \\
\hline & $3-3$ & $5599.50 \mathrm{M} 1$ & $8.43(-2)$ \\
\hline & $2-3$ & 5724.35 M1 & $6.46(-2)$ \\
\hline
\end{tabular}

Table 3. continued

\begin{tabular}{|c|c|c|c|}
\hline \multicolumn{2}{|c|}{ Multiplet $J-J^{\prime}$} & \multirow{2}{*}{$\frac{\lambda(\AA)^{a} \text { Type }}{5123.15 \text { M1,E2 }}$} & \multirow{2}{*}{$\frac{A_{k i}}{9.18(-3)}$} \\
\hline \multirow[t]{5}{*}{$a^{5} F-b^{3} P$} & $3-2$ & & \\
\hline & $2-2$ & 5227.47 M1,E2 & $1.33(-3)^{*}$ \\
\hline & $2-1$ & 5175.25 M1,E2 & $2.97(-3)$ \\
\hline & $1-2$ & $5298.00 \mathrm{M} 1$ & $1.15(-3)$ \\
\hline & $1-1$ & 5244.37 M1,E2 & $1.60(-3)$ \\
\hline \multirow[t]{5}{*}{$a^{5} \mathrm{~F}-\mathrm{c}^{3} \mathrm{P}$} & $3-2$ & 4918.49 M1,E2 & $3.98(-2)$ \\
\hline & $2-2$ & 5014.56 M1,E2 & $5.30(-3)^{*}$ \\
\hline & $2-1$ & 4908.42 M1 & $3.63(-2)$ \\
\hline & $1-2$ & 5079.43 M1,E2 & $4.60(-3)$ \\
\hline & $1-1$ & 4970.56 M1,E2 & $2.57(-2)$ \\
\hline \multirow[t]{3}{*}{$a^{5} \mathrm{~F}-\mathrm{a}^{3} \mathrm{H}$} & $5-5$ & 4145.02 M1,E2 & $2.05((-3)$ \\
\hline & $4-5$ & 4264.99 M1,E2 & $1.03(-3)$ \\
\hline & $4-4$ & 4187.59 M1,E2 & $1.08(-3)^{*}$ \\
\hline \multirow[t]{15}{*}{$a^{5} F-a^{5} D$} & $5-4$ & $2676.94 \mathrm{E} 2$ & $1.42(+1)$ \\
\hline & $5-3$ & $2633.32 \mathrm{E} 2$ & $6.64(+0)$ \\
\hline & $4-4$ & $2726.47 \mathrm{E} 2$ & $5.61(+0)$ \\
\hline & $4-3$ & $2681.23 \mathrm{E} 2$ & $4.99(+0)$ \\
\hline & $4-2$ & $2651.08 \mathrm{E} 2$ & $1.06(+1)$ \\
\hline & $3-4$ & $2766.60 \mathrm{E} 2$ & $1.12(+0)$ \\
\hline & $3-3$ & $2720.03 \mathrm{E} 2$ & $7.14(+0)$ \\
\hline & $3-2$ & $2689.00 \mathrm{E} 2$ & $9.22(-1)$ \\
\hline & $3-1$ & $2669.57 \mathrm{E} 2$ & $1.34(+1)$ \\
\hline & $2-4$ & $2796.74 \mathrm{E} 2$ & $9.24(-2)$ \\
\hline & $2-3$ & $2749.16 \mathrm{E} 2$ & $2.49(+0)$ \\
\hline & $2-2$ & $2717.47 \mathrm{E} 2$ & $6.70(+0)$ \\
\hline & $1-3$ & $2768.55 \mathrm{E} 2$ & $2.30(-1)$ \\
\hline & $1-2$ & $2736.41 \mathrm{E} 2$ & $3.42(+0)$ \\
\hline & $1-1$ & $2716.29 \mathrm{E} 2$ & $8.27(+0)$ \\
\hline \multirow[t]{8}{*}{$a^{5} F-a^{3} D$} & $4-3$ & 4262.17 M1 & $5.36(-1)$ \\
\hline & $3-3$ & 4361.05 M1 & $1.20(-2)$ \\
\hline & $3-2$ & $4244.86 \mathrm{M} 1$ & $6.98(-1)$ \\
\hline & $2-3$ & 4436.41 M1 & $2.53(-2)$ \\
\hline & $2-2$ & 4316.23 M1 & $6.10(-2)^{*}$ \\
\hline & $2-1$ & $4110.89 \mathrm{M} 1$ & $3.02(-1)$ \\
\hline & $1-2$ & 4364.21 M1 & $6.34(-2)$ \\
\hline & $1-1$ & 4154.39 M1 & $1.41(-1)$ \\
\hline \multirow[t]{2}{*}{$a^{5} \mathrm{~F}-\mathrm{a}^{1} \mathrm{P}$} & $2-1$ & 4416.68 M1 & $3.60(-1)$ \\
\hline & $1-1$ & $4466.92 \mathrm{M} 1$ & $1.65(-1)$ \\
\hline$a^{5} F-b^{1} D$ & $3-2$ & $3752.87 \mathrm{M} 1$ & $5.90(-3)$ \\
\hline \multirow[t]{11}{*}{$a^{5} F-c^{3} F$} & $5-3$ & $2663.82 \mathrm{E} 2$ & $1.94(-3)$ \\
\hline & $5-4$ & 2651.98 M1,E2 & $2.67(-1)$ \\
\hline & $4-3$ & 2712.87 M1,E2 & $3.87(-2)$ \\
\hline & $4-4$ & 2700.58 M1,E2 & $7.94(-2)^{*}$ \\
\hline & $3-2$ & $2760.83 \mathrm{M} 1$ & $5.86(-3)$ \\
\hline & $3-3$ & 2752.59 M1,E2 & $3.30(-2)^{*}$ \\
\hline & $3-4$ & 2739.95 M1,E2 & $2.93(-2)$ \\
\hline & $2-2$ & $2790.84 \mathrm{M} 1$ & $3.02(-2)$ \\
\hline & $2-3$ & $2782.43 \mathrm{M} 1, \mathrm{E} 2$ & $6.37(-2)$ \\
\hline & $1-2$ & $2810.82 \mathrm{M} 1, \mathrm{E} 2$ & $1.03(-1)$ \\
\hline & $1-3$ & 2802.29 E2 & $1.28(-3)$ \\
\hline \multirow[t]{2}{*}{$b^{3} F-b^{3} F$} & $4-3$ & $111637.78 \mathrm{M} 1$ & $1.67(-2)$ \\
\hline & $3-2$ & $162957.42 \mathrm{M} 1$ & $7.26(-3)$ \\
\hline \multirow[t]{2}{*}{$b^{3} F-a^{1} G$} & $4-4$ & 10661.30 M1 & $6.89(-3)$ \\
\hline & $3-4$ & $11786.95 \mathrm{M} 1$ & $2.82(-3)$ \\
\hline \multirow[t]{5}{*}{$b^{3} F-a^{3} G$} & $4-5$ & $8463.87 \mathrm{M} 1$ & $3.57(-2)$ \\
\hline & $4-4$ & 8196.81 M1 & $4.33(-2)^{*}$ \\
\hline & $4-3$ & 7933.29 M1 & $3.06(-3)$ \\
\hline & $3-3$ & 8540.19 M1 & $3.79(-2)$ \\
\hline & $2-3$ & $9012.51 \mathrm{M} 1$ & $2.27(-2)$ \\
\hline
\end{tabular}


Table 3. continued

\begin{tabular}{|c|c|c|c|}
\hline Multiplet & $J-J^{\prime}$ & $\lambda(\AA)^{a}$ Type & $A_{k i}$ \\
\hline \multirow[t]{5}{*}{$b^{3} \mathrm{~F}-\mathrm{b}^{3} \mathrm{P}$} & $4-2$ & $7009.88 \mathrm{E} 2$ & $3.02(-2)$ \\
\hline & $3-2$ & $7479.54 \mathrm{E} 2$ & $5.74(-3)$ \\
\hline & $3-1$ & $7373.09 \mathrm{E} 2$ & $3.04(-2)$ \\
\hline & $2-1$ & $7722.50 \mathrm{M} 1, \mathrm{E} 2$ & $2.30(-2)$ \\
\hline & $2-0$ & $7637.67 \mathrm{E} 2$ & $2.74(-2)$ \\
\hline \multirow[t]{5}{*}{$b^{3} F-c^{3} P$} & $4-2$ & $6632.28 \mathrm{E} 2$ & $2.04(-2)$ \\
\hline & $3-2$ & 7051.19 M1,E2 & $1.03(-2)$ \\
\hline & $2-2$ & $7370.09 \mathrm{M} 1$ & $1.63(-2)$ \\
\hline & $2-1$ & $7143.09 \mathrm{M} 1, \mathrm{E} 2$ & $4.37(-3)$ \\
\hline & $2-0$ & $6875.85 \mathrm{E} 2$ & $1.53(-3)$ \\
\hline \multirow[t]{3}{*}{$b^{3} F-b^{1} G$} & $4-4$ & $6519.48 \mathrm{M} 1$ & $1.84(-1)$ \\
\hline & $3-4$ & 6923.83 M1 & $1.06(-1)$ \\
\hline & $2-4$ & $7231.07 \mathrm{E} 2$ & $2.48(-3)$ \\
\hline \multirow[t]{2}{*}{$b^{3} \mathrm{~F}-\mathrm{a}^{3} \mathrm{H}$} & $4-4$ & $5526.56 \mathrm{M} 1$ & $7.44(-3)^{*}$ \\
\hline & $3-4$ & $5814.40 \mathrm{M} 1$ & $3.87(-3)$ \\
\hline \multirow[t]{7}{*}{$b^{3} F-a^{3} D$} & $4-3$ & $5657.21 \mathrm{M} 1$ & $1.07(-1)$ \\
\hline & $3-3$ & $5959.20 \mathrm{M} 1$ & $6.24(-2)^{*}$ \\
\hline & $3-2$ & 5744.35 M1 & $8.88(-3)$ \\
\hline & $3-1$ & $5386.29 \mathrm{E} 2$ & $2.25(-3)$ \\
\hline & $2-3$ & 6185.39 M1,E2 & $7.66(-3)$ \\
\hline & $2-2$ & $5954.24 \mathrm{M} 1$ & $3.44(-2)$ \\
\hline & $2-1$ & $5570.41 \mathrm{M} 1, \mathrm{E} 2$ & $4.43(-2)$ \\
\hline \multirow[t]{2}{*}{$b^{3} \mathrm{~F}-\mathrm{a}^{1} \mathrm{P}$} & $3-1$ & $5923.64 \mathrm{E} 2$ & $3.83(-3)$ \\
\hline & $2-1$ & 6147.09 M1,E2 & $3.00(-2)$ \\
\hline \multirow[t]{2}{*}{$b^{3} F-b^{1} D$} & $3-2$ & $4878.82 \mathrm{M} 1$ & $5.38(-1)$ \\
\hline & $2-2$ & $5029.40 \mathrm{M} 1, \mathrm{E} 2$ & $3.12(-1)$ \\
\hline \multirow[t]{5}{*}{$b^{3} F-a^{5} D$} & $4-3$ & $3173.56 \mathrm{E} 2$ & $6.70(-3)$ \\
\hline & $3-3$ & $3266.42 \mathrm{M} 1, \mathrm{E} 2$ & $1.06(-3)^{*}$ \\
\hline & $3-2$ & $3221.78 \mathrm{E} 2$ & $4.44(-3)$ \\
\hline & $2-2$ & 3286.76 M1,E2 & $1.45(-3)$ \\
\hline & $2-1$ & $3257.78 \mathrm{M} 1, \mathrm{E} 2$ & $2.62(-3)$ \\
\hline \multirow[t]{7}{*}{$b^{3} F-c^{3} F$} & $4-3$ & $3217.97 \mathrm{M} 1, \mathrm{E} 2$ & $1.59(-2)$ \\
\hline & $4-4$ & $3200.70 \mathrm{M} 1, \mathrm{E} 2$ & $3.24(-3)^{*}$ \\
\hline & $3-2$ & $3325.43 \mathrm{M} 1, \mathrm{E} 2$ & $2.18(-2)$ \\
\hline & $3-3$ & $3313.49 \mathrm{E} 2$ & $2.29(-3)$ \\
\hline & $3-4$ & 3295.18 M1 & $2.00(-2)$ \\
\hline & $2-3$ & $3382.27 \mathrm{M} 1, \mathrm{E} 2$ & $2.43(-2)$ \\
\hline & $2-4$ & $3363.19 \mathrm{E} 2$ & $1.21(-2)$ \\
\hline \multirow[t]{2}{*}{$b^{3} \mathrm{~F}-\mathrm{a}^{1} \mathrm{~F}$} & $3-3$ & 2994.71 M1 & $9.77(-3)^{*}$ \\
\hline & $2-3$ & $3050.78 \mathrm{M} 1, \mathrm{E} 2$ & $1.69(-1)$ \\
\hline \multirow[t]{2}{*}{$a^{1} D-a^{3} P$} & $2-2$ & $62122.27 \mathrm{M} 1$ & $2.96(-2)$ \\
\hline & $2-1$ & $57029.52 \mathrm{M} 1$ & $2.55(-2)$ \\
\hline$a^{1} D-a^{1} G$ & $2-4$ & $13261.38 \mathrm{E} 2$ & $1.80(-3)$ \\
\hline \multirow{3}{*}{$a^{1} \mathrm{D}-\mathrm{b}^{3} \mathrm{P}$} & $2-2$ & 8047.29 M1,E2 & $8.56(-3)^{*}$ \\
\hline & $2-1$ & 7924.21 E2 & $5.20(-2)$ \\
\hline & $2-0$ & $7834.91 \mathrm{E} 2$ & $6.39(-2)$ \\
\hline \multirow[t]{3}{*}{$a^{1} D-c^{3} P$} & $2-2$ & $7553.59 \mathrm{M} 1, \mathrm{E} 2$ & $2.68(-2)^{*}$ \\
\hline & $2-1$ & 7315.32 M1,E2 & $7.23(-3)^{*}$ \\
\hline & $2-0$ & $7035.30 \mathrm{E} 2$ & $1.18(-3)$ \\
\hline$a^{1} D-b^{1} G$ & $2-4$ & $7407.62 \mathrm{E} 2$ & $7.14(-2)$ \\
\hline$a^{1} D-a^{3} H$ & $2-4$ & $6151.81 \mathrm{E} 2$ & $1.44(-2)$ \\
\hline \multirow[t]{3}{*}{$a^{1} D-a^{3} D$} & $2-3$ & 6314.12 M1,E2 & $6.24(-3)$ \\
\hline & $2-2$ & $6073.44 \mathrm{M} 1, \mathrm{E} 2$ & $3.92(-3)^{*}$ \\
\hline & $2-1$ & $5674.60 \mathrm{E} 2$ & $3.29(-1)$ \\
\hline$a^{1} D-a^{1} P$ & $2-1$ & $6274.22 \mathrm{E} 2$ & $1.67(-1)$ \\
\hline$a^{1} D-b^{1} D$ & $2-2$ & $5114.18 \mathrm{M} 1, \mathrm{E} 2$ & $9.88(-1)$ \\
\hline$a^{1} D-a^{5} D$ & $2-4$ & $3442.05 \mathrm{E} 2$ & $2.72(-3)$ \\
\hline
\end{tabular}

Table 3. continued

\begin{tabular}{|c|c|c|c|}
\hline \multicolumn{2}{|c|}{ Multiplet $J-J^{\prime}$} & \multirow{2}{*}{$\frac{\lambda(\AA)^{a} \text { Type }}{3433.13 \text { E2 }}$} & \multirow{2}{*}{$\begin{array}{l}A_{k i} \\
4.80(-2)\end{array}$} \\
\hline$a^{1} D-c^{3} F$ & $2-2$ & & \\
\hline & $2-3$ & $3420.40 \mathrm{E} 2$ & $2.57(-1)$ \\
\hline & $2-4$ & $3400.90 \mathrm{E} 2$ & $5.41(-1)$ \\
\hline$a^{1} D-a^{1} F$ & $2-3$ & $3081.77 \mathrm{E} 2$ & $3.93(+0)$ \\
\hline \multirow[t]{3}{*}{$a^{3} \mathrm{P}-\mathrm{a}^{5} \mathrm{P}$} & $2-3$ & $22162.28 \mathrm{M} 1$ & $1.04(-3)$ \\
\hline & $2-2$ & $20954.77 \mathrm{M} 1$ & $1.53(-3)^{*}$ \\
\hline & $1-1$ & 20260.69 M1 & $2.89(-3)$ \\
\hline \multirow[t]{6}{*}{$a^{3} \mathrm{P}-\mathrm{b}^{3} \mathrm{P}$} & $2-2$ & $9244.88 \mathrm{E} 2$ & $9.36(-3)$ \\
\hline & $2-1$ & $9082.80 \mathrm{M} 1, \mathrm{E} 2$ & $3.90(-2)$ \\
\hline & $2-0$ & $8965.67 \mathrm{E} 2$ & $7.37(-2)$ \\
\hline & $1-2$ & $9369.40 \mathrm{M} 1, \mathrm{E} 2$ & $1.91(-2)^{*}$ \\
\hline & $1-1$ & 9202.96 M1,E2 & $1.83(-2)$ \\
\hline & $0-2$ & $9538.20 \mathrm{E} 2$ & $7.98(-3)$ \\
\hline \multirow[t]{5}{*}{$a^{3} \mathrm{P}-c^{3} \mathrm{P}$} & $2-2$ & 8599.20 M1,E2 & $1.74(-2)^{*}$ \\
\hline & $2-1$ & 8291.74 M1,E2 & $2.21(-3)^{*}$ \\
\hline & $1-2$ & $8706.82 \mathrm{E} 2$ & $2.48(-2)$ \\
\hline & $1-1$ & 8391.77 M1 & $1.06(-3)^{*}$ \\
\hline & $0-2$ & 8852.42 E2 & $7.86(-3)$ \\
\hline$a^{3} P-b^{1} G$ & $2-4$ & $8410.52 \mathrm{E} 2$ & $1.17(-2)$ \\
\hline$a^{3} \mathrm{P}-\mathrm{a}^{3} \mathrm{H}$ & $2-4$ & $6827.97 \mathrm{E} 2$ & $2.53(-3)$ \\
\hline \multirow[t]{7}{*}{$a^{3} \mathrm{P}-\mathrm{a}^{3} \mathrm{D}$} & $2-3$ & $7028.51 \mathrm{M} 1, \mathrm{E} 2$ & $1.83(-2)$ \\
\hline & $2-2$ & $6731.56 \mathrm{M} 1, \mathrm{E} 2$ & $2.06(-2)$ \\
\hline & $2-1$ & 6245.07 M1,E2 & $1.70(-1)$ \\
\hline & $1-3$ & $7100.25 \mathrm{E} 2$ & $1.11(-2)$ \\
\hline & $1-2$ & 6797.34 M1,E2 & $5.86(-3)$ \\
\hline & $1-1$ & $6301.64 \mathrm{M} 1, \mathrm{E} 2$ & $1.02(-2)^{*}$ \\
\hline & $0-2$ & $6885.75 \mathrm{E} 2$ & $1.57(-2)$ \\
\hline \multirow[t]{3}{*}{$a^{3} \mathrm{P}-\mathrm{a}^{1} \mathrm{P}$} & $2-1$ & 6979.10 M1,E2 & $5.33(-2)$ \\
\hline & $1-1$ & 7049.83 M1,E2 & $3.37(-2)^{*}$ \\
\hline & $0-1$ & $7144.98 \mathrm{M} 1$ & $2.53(-3)^{*}$ \\
\hline \multirow[t]{3}{*}{$a^{3} P-b^{1} D$} & $2-2$ & $5572.98 \mathrm{E} 2$ & $2.06(-1)$ \\
\hline & $1-2$ & $5617.98 \mathrm{M} 1, \mathrm{E} 2$ & $4.40(-3)^{*}$ \\
\hline & $0-2$ & $5678.24 \mathrm{E} 2$ & $4.50(-3)$ \\
\hline \multirow[t]{6}{*}{$a^{3} P-a^{5} D$} & $2-4$ & $3643.96 \mathrm{E} 2$ & $7.50(-3)$ \\
\hline & $2-3$ & $3563.61 \mathrm{M} 1, \mathrm{E} 2$ & $2.19(-3)$ \\
\hline & $2-1$ & 3477.49 M1,E2 & $1.64(-3)^{*}$ \\
\hline & $1-3$ & $3581.96 \mathrm{E} 2$ & $2.99(-3)$ \\
\hline & $1-2$ & 3528.34 M1,E2 & $3.30(-3)$ \\
\hline & $0-1$ & $3518.19 \mathrm{M} 1$ & $1.87(-3)$ \\
\hline \multirow[t]{6}{*}{$a^{3} \mathrm{P}-c^{3} \mathrm{~F}$} & $2-2$ & $3633.96 \mathrm{E} 2$ & $1.14(-1)$ \\
\hline & $2-3$ & $3619.71 \mathrm{E} 2$ & $5.26(-1)$ \\
\hline & $2-4$ & $3597.87 \mathrm{E} 2$ & $1.70(+0)$ \\
\hline & $1-2$ & $3653.04 \mathrm{E} 2$ & $9.04(-1)$ \\
\hline & $1-3$ & $3638.64 \mathrm{E} 2$ & $1.25(+0)$ \\
\hline & $0-2$ & $3678.43 \mathrm{E} 2$ & $8.20(-1)$ \\
\hline \multirow[t]{2}{*}{$a^{3} \mathrm{P}-\mathrm{a}^{1} \mathrm{~F}$} & $2-3$ & $3242.64 \mathrm{E} 2$ & $8.79(-1)$ \\
\hline & $1-3$ & 3257.83 E2 & $8.10(-3)$ \\
\hline$a^{5} \mathrm{P}-\mathrm{a}^{5} \mathrm{P}$ & $2-1$ & $325485.09 \mathrm{M} 1$ & $1.18(-3)$ \\
\hline \multirow[t]{6}{*}{$a^{5} \mathrm{P}-\mathrm{b}^{3} \mathrm{P}$} & $3-2$ & 15861.40 M1 & $4.84(-3)$ \\
\hline & $2-2$ & $16543.69 \mathrm{M} 1$ & $2.00(-3)^{*}$ \\
\hline & $2-1$ & $16031.75 \mathrm{M} 1$ & $1.03(-3)$ \\
\hline & $1-2$ & $17429.60 \mathrm{M} 1$ & $1.18(-3)$ \\
\hline & $1-1$ & $16862.30 \mathrm{M} 1$ & $2.44(-2)$ \\
\hline & $1-0$ & $16463.02 \mathrm{M} 1$ & $4.75(-2)$ \\
\hline \multirow[t]{5}{*}{$a^{5} \mathrm{P}-\mathrm{c}^{3} \mathrm{P}$} & $3-2$ & 14051.25 M1 & $1.72(-1)$ \\
\hline & $2-2$ & $14584.08 \mathrm{M} 1$ & $9.32(-2)$ \\
\hline & $1-2$ & $15268.20 \mathrm{M} 1$ & $1.93(-2)$ \\
\hline & $1-1$ & $14325.09 \mathrm{M} 1$ & $1.42(-1)$ \\
\hline & $1-0$ & 13289.29 M1 & $1.49(-1)$ \\
\hline
\end{tabular}


Table 3. continued

\begin{tabular}{|c|c|c|c|}
\hline Multiplet & $J-J^{\prime}$ & $\lambda(\AA)^{a}$ Type & $A_{k i}$ \\
\hline \multirow[t]{5}{*}{$a^{5} \mathrm{P}-\mathrm{a}^{3} \mathrm{D}$} & $3-3$ & 10292.77 M1 & $4.34(-2)$ \\
\hline & $2-3$ & $10575.80 \mathrm{M} 1$ & $1.21(-2)$ \\
\hline & $2-2$ & 9917.51 M1 & $4.02(-2)$ \\
\hline & $1-2$ & $10229.20 \mathrm{M} 1$ & $1.07(-2)$ \\
\hline & $1-1$ & 9146.48 M1 & $4.70(-3)$ \\
\hline \multirow[t]{2}{*}{$\mathrm{a}^{5} \mathrm{P}-\mathrm{a}^{1} \mathrm{P}$} & $2-1$ & $10464.33 \mathrm{M} 1$ & $1.49(-3)$ \\
\hline & $1-1$ & 10811.93 M1 & $2.46(-2)$ \\
\hline$a^{5} \mathrm{P}-\mathrm{b}^{1} \mathrm{D}$ & $3-2$ & $7445.18 \mathrm{M} 1$ & $2.48(-3)$ \\
\hline \multirow[t]{10}{*}{$a^{5} \mathrm{P}-\mathrm{a}^{5} \mathrm{D}$} & $3-4$ & $4361.04 \mathrm{E} 2$ & $5.13(-1)$ \\
\hline & $3-3$ & $4246.44 \mathrm{E} 2$ & $6.36(-1)$ \\
\hline & $3-2$ & $4171.30 \mathrm{E} 2$ & $4.60(-1)$ \\
\hline & $3-1$ & $4124.73 \mathrm{E} 2$ & $1.88(-1)$ \\
\hline & $2-4$ & $4411.05 \mathrm{E} 2$ & $3.92(-1)$ \\
\hline & $2-2$ & $4217.04 \mathrm{E} 2$ & $2.72(-1)$ \\
\hline & $2-1$ & $4169.44 \mathrm{E} 2$ & $7.97(-1)$ \\
\hline & $1-3$ & $4351.26 \mathrm{E} 2$ & $3.70(-1)$ \\
\hline & $1-2$ & $4272.39 \mathrm{E} 2$ & $3.56(-1)$ \\
\hline & $1-1$ & $4223.55 \mathrm{E} 2$ & $1.62(-1)$ \\
\hline \multirow[t]{5}{*}{$a^{5} \mathrm{P}-c^{3} \mathrm{~F}$} & $3-3$ & $4326.34 \mathrm{M} 1, \mathrm{E} 2$ & $1.22(-3)$ \\
\hline & $3-4$ & 4295.18 M1,E2 & $3.50(-3)$ \\
\hline & $2-3$ & $4375.56 \mathrm{E} 2$ & $1.93(-3)$ \\
\hline & $1-2$ & $4456.60 \mathrm{M} 1, \mathrm{E} 2$ & $1.65(-3)$ \\
\hline & $1-3$ & 4435 & $3.27(-3)$ \\
\hline$a^{1} G-b^{1} G$ & $4-4$ & 16781.66 M1,E2 & $2.76(-3)$ \\
\hline \multirow{2}{*}{$a^{1} G-a^{3} H$} & $4-5$ & 12075.42 M1,E2 & $3.58(-3)$ \\
\hline & $4-4$ & 11474.94 M1,E2 & $5.19(-3)^{*}$ \\
\hline$a^{1} G-a^{1} H$ & $4-5$ & $8787.06 \mathrm{E} 2$ & $1.19(-1)$ \\
\hline$a^{1} G-b^{1} D$ & $4-2$ & $8324.52 \mathrm{E} 2$ & $1.61(-2)$ \\
\hline \multirow[t]{2}{*}{$a^{1} G-c^{3} F$} & $4-3$ & 4609.27 M1,E2 & $8.06(-3)$ \\
\hline & $4-4$ & $4573.92 \mathrm{M} 1, \mathrm{E} 2$ & $4.28(-3)^{*}$ \\
\hline$a^{1} G-a^{1} F$ & $4-3$ & $4014.80 \mathrm{E} 2$ & $3.99(+0)$ \\
\hline \multirow[t]{2}{*}{$a^{3} G-a^{3} G$} & $5-4$ & 259784.18 M1 & $1.27(-3)$ \\
\hline & $4-3$ & 246767 & $1.70(-3)$ \\
\hline \multirow[t]{3}{*}{$a^{3} G-b^{1} G$} & $5-4$ & 28379.33 M1 & $1.44(-2)$ \\
\hline & $4-4$ & 3185 & $1.86(-3)^{*}$ \\
\hline & $3-4$ & $36582.93 \mathrm{M} 1$ & $1.05(-2)$ \\
\hline \multirow[t]{6}{*}{$a^{3} G-a^{3} H$} & $5-6$ & $18238.80 \mathrm{M} 1$ & $4.18(-2)$ \\
\hline & $5-5$ & 17105.46 M1 & $4.14(-2)^{*}$ \\
\hline & $5-4$ & 15924. & $5.34(-3)$ \\
\hline & $4-5$ & 18311.16 M1,E2 & $1.97(-3)$ \\
\hline & $4-4$ & 16964.94 M1 & $7.16(-2)$ \\
\hline & $3-4$ & 18217.36 M1 & $3.19(-2)$ \\
\hline \multirow[t]{2}{*}{$a^{3} G-a^{1} H$} & $5-5$ & 11179.22 M1 & $1.10(-1)^{*}$ \\
\hline & $4-5$ & 11681.93 M1 & $4.66(-2)$ \\
\hline$a^{3} G-a^{5} D$ & $5-4$ & $5242.12 \mathrm{M} 1, \mathrm{E} 2$ & $1.19(-3)$ \\
\hline \multirow[t]{8}{*}{$a^{3} G-c^{3} F$} & $5-3$ & $5192.06 \mathrm{E} 2$ & $1.79(-2)$ \\
\hline & $5-4$ & 5147.26 M1,E2 & $1.19(-1)$ \\
\hline & $4-2$ & $5328.54 \mathrm{E} 2$ & $1.97(-2)$ \\
\hline & $4-3$ & 5297.95 M1,E2 & $2.79(-2)$ \\
\hline & $4-4$ & $5251.30 \mathrm{M} 1, \mathrm{E} 2$ & $4.86(-2)^{*}$ \\
\hline & $3-2$ & 5446.14 M1,E2 & $1.01(-1)$ \\
\hline & & $5414.19 \mathrm{M} 1, \mathrm{E} 2$ & $7.07(-2)$ \\
\hline & $3-4$ & $5365.49 \mathrm{M} 1, \mathrm{E} 2$ & $3.69(-3)$ \\
\hline \multirow[t]{3}{*}{$a^{3} G-a^{1} F$} & $5-3$ & $4449.86 \mathrm{E} 2$ & $1.90(-3)$ \\
\hline & & 4527.41 M1,E2 & $1.09(-1)$ \\
\hline & $3-3$ & $4612.03 \mathrm{M} 1$ & $6.89(-2)$ \\
\hline \multirow[t]{3}{*}{$b^{3} \mathrm{P}-\mathrm{c}^{3} \mathrm{P}$} & $2-1$ & 80425.36 M1 & $2.86(-2)$ \\
\hline & $1-2$ & 161506.86 M1 & $3.02(-3)$ \\
\hline & $1-0$ & 62716.98 M1 & $8.84(-3)$ \\
\hline
\end{tabular}

Table 3. continued

\begin{tabular}{|c|c|c|c|}
\hline \multicolumn{2}{|c|}{ Multiplet $J-J^{\prime}$} & \multirow{2}{*}{$\frac{\lambda(\AA)^{a} \text { Type }}{29317.43 \mathrm{M} 1}$} & \multirow{2}{*}{$\frac{A_{k i}}{8.63(-3)}$} \\
\hline$b^{3} P-a^{3} D$ & $2-3$ & & \\
\hline & $2-2$ & 24761.29 M1 & $1.39(-2)$ \\
\hline & $2-1$ & $19246.36 \mathrm{M} 1$ & $2.05(-2)$ \\
\hline & $1-2$ & 26004.16 M1 & $1.63(-3)$ \\
\hline & $1-1$ & 19988.94 M1 & $1.23(-2)^{*}$ \\
\hline & $0-1$ & $20580.64 \mathrm{M} 1$ & $2.35(-2)$ \\
\hline \multirow[t]{3}{*}{$b^{3} \mathrm{P}-\mathrm{a}^{1} \mathrm{P}$} & $2-1$ & $28476.50 \mathrm{M} 1$ & $2.39(-2)$ \\
\hline & $1-1$ & 30132.78 M1 & $3.08(-2)$ \\
\hline & $0-1$ & 31497.89 M1 & $3.22(-2)$ \\
\hline$b^{3} \mathrm{P}-\mathrm{b}^{1} \mathrm{D}$ & $1-2$ & $14422.01 \mathrm{M} 1$ & $1.79(-2)$ \\
\hline \multirow[t]{5}{*}{$b^{3} \mathrm{P}-\mathrm{c}^{3} \mathrm{~F}$} & $2-2$ & $5987.62 \mathrm{M} 1, \mathrm{E} 2$ & $2.02(-3)$ \\
\hline & $2-3$ & 5949.02 M1,E2 & $3.26(-3)$ \\
\hline & $2-4$ & $5890.27 \mathrm{E} 2$ & $3.81(-3)$ \\
\hline & $1-2$ & $6057.63 \mathrm{E} 2$ & $1.62(-3)$ \\
\hline & $0-2$ & $6110.87 \mathrm{E} 2$ & $2.28(-3)$ \\
\hline$b^{3} \mathrm{P}-\mathrm{a}^{1} \mathrm{~F}$ & $1-3$ & $5043.14 \mathrm{E} 2$ & $2.30(-3)$ \\
\hline$c^{3} \mathrm{P}-\mathrm{c}^{3} \mathrm{P}$ & $1-0$ & $183790.32 \mathrm{M} 1$ & $5.46(-3)$ \\
\hline \multirow[t]{6}{*}{$c^{3} \mathrm{P}-\mathrm{a}^{3} \mathrm{D}$} & $2-3$ & $38480.06 \mathrm{M} 1$ & $1.24(-2)$ \\
\hline & $2-2$ & 30994.59 M1 & $1.88(-2)$ \\
\hline & $2-1$ & 22812.32 M1 & $5.63(-2)$ \\
\hline & $1-2$ & 35775.97 M1 & $1.17(-3)$ \\
\hline & $1-1$ & 25301.09 M1 & $5.33(-2)$ \\
\hline & $0-1$ & 29340.14 M1 & $1.18(-2)$ \\
\hline \multirow[t]{3}{*}{$c^{3} \mathrm{P}-\mathrm{a}^{1} \mathrm{P}$} & $2-1$ & $37044.23 \mathrm{M} 1$ & $1.35(-3)$ \\
\hline & $1-1$ & 44086.30 M1 & $1.75(-2)$ \\
\hline & $0-1$ & 57998.59 M1 & $2.32(-3)$ \\
\hline \multirow[t]{2}{*}{$c^{3} \mathrm{P}-\mathrm{b}^{1} \mathrm{D}$} & $2-2$ & $15836.13 \mathrm{M} 1$ & $1.36(-1)$ \\
\hline & $1-2$ & 16996.75 M1 & $1.02(-2)$ \\
\hline \multirow[t]{6}{*}{$c^{3} \mathrm{P}-\mathrm{c}^{3} \mathrm{~F}$} & $2-2$ & $6293.69 \mathrm{M} 1, \mathrm{E} 2$ & $4.64(-3)^{*}$ \\
\hline & $2-3$ & $6251.06 \mathrm{M} 1, \mathrm{E} 2$ & $1.46(-2)$ \\
\hline & $2-4$ & $6186.22 \mathrm{E} 2$ & $1.67(-2)$ \\
\hline & $1-2$ & 6469.25 M1,E2 & $1.67(-2)$ \\
\hline & $1-3$ & $6424.22 \mathrm{E} 2$ & $8.60(-3)$ \\
\hline & $0-2$ & $6705.28 \mathrm{E} 2$ & $6.96(-3)$ \\
\hline \multirow[t]{2}{*}{$b^{1} G-a^{3} H$} & $4-5$ & 43059.03 M1 & $1.65(-2)$ \\
\hline & $4-4$ & 36287.74 M1 & $1.78(-2)$ \\
\hline$b^{1} G-a^{1} H$ & $4-5$ & 18445.18 M1,E2 & $3.59(-3)$ \\
\hline \multirow[t]{3}{*}{$b^{1} G-c^{3} F$} & $4-2$ & $6398.75 \mathrm{E} 2$ & $2.56(-3)$ \\
\hline & $4-3$ & 6354.69 M1,E2 & $4.29(-2)$ \\
\hline & $4-4$ & $6287.69 \mathrm{M} 1$ & $6.49(-2)$ \\
\hline$b^{1} G-a^{1} F$ & $4-3$ & $5277.37 \mathrm{M} 1, \mathrm{E} 2$ & $1.27(-3)^{*}$ \\
\hline$a^{3} H-a^{3} H$ & $5-4$ & $230756.04 \mathrm{M} 1$ & $1.88(-3)$ \\
\hline \multirow[t]{2}{*}{$a^{3} \mathrm{H}-\mathrm{a}^{1} \mathrm{H}$} & $6-5$ & 28882.17 M1 & $1.11(-2)$ \\
\hline & $4-5$ & 37513.36 M1 & $5.74(-3)$ \\
\hline \multirow[t]{5}{*}{$a^{3} \mathrm{H}-c^{3} \mathrm{~F}$} & $6-4$ & $7171.06 \mathrm{E} 2$ & $3.57(-2)$ \\
\hline & $5-3$ & $7454.90 \mathrm{E} 2$ & $2.70(-2)$ \\
\hline & $5-4$ & 7362.86 M1,E2 & $3.72(-3)$ \\
\hline & $4-2$ & $7768.63 \mathrm{E} 2$ & $2.42(-2)$ \\
\hline & $4-3$ & 7703.78 M1,E2 & $4.20(-3)$ \\
\hline$a^{3} H-a^{1} F$ & $5-3$ & $6014.53 \mathrm{E} 2$ & $2.21(-3)$ \\
\hline \multirow[t]{2}{*}{$a^{3} D-a^{3} D$} & $3-2$ & 159331.81 M1 & $4.42(-3)$ \\
\hline & $2-1$ & 86413.59 M1 & $2.74(-2)$ \\
\hline \multirow[t]{3}{*}{$a^{3} D-b^{1} D$} & $3-2$ & 26911.21 M1 & $2.50(-2)$ \\
\hline & $2-2$ & $32380.25 \mathrm{M} 1$ & $6.74(-3)$ \\
\hline & $1-2$ & $51784.59 \mathrm{M} 1$ & $1.68(-3)$ \\
\hline
\end{tabular}


Table 3. continued

\begin{tabular}{|c|c|c|c|}
\hline \multicolumn{2}{|c|}{ Multiplet $J-J^{\prime}$} & \multicolumn{2}{|c|}{$\lambda(\AA)^{a}$ Type $A_{k i}$} \\
\hline \multirow[t]{7}{*}{$a^{3} D-c^{3} F$} & $3-2$ & $7524.36 \mathrm{M} 1, \mathrm{E} 2$ & $1.52(-3)$ \\
\hline & $3-3$ & & $1.06(-2)^{*}$ \\
\hline & $3-4$ & $7 \mathrm{M} 1, \mathrm{E} 2$ & $2.01(-2)$ \\
\hline & $2-2$ & 7897.31 M1,E2 & $1.20(-2)$ \\
\hline & $2-3$ & 7830 & $4.24(-3)$ \\
\hline & & 7728 & $4.76(-3)$ \\
\hline & & I1,E2 & $2.90(-3)$ \\
\hline \multirow[t]{3}{*}{$a^{3} D-a^{1} F$} & $3-3$ & 6020.13 M1,E2 & $8.23(-3)^{*}$ \\
\hline & $2-3$ & $6256.53 \mathrm{M} 1, \mathrm{E} 2$ & $6.09(-3)$ \\
\hline & $1-3$ & $6744.88 \mathrm{E} 2$ & $5.19(-3)$ \\
\hline \multirow[t]{2}{*}{$a^{1} P-a^{3} D$} & $1-2$ & 189791.64 M1 & $1.09(-3)$ \\
\hline & $1-1$ & 5937 & $1.24(-2)$ \\
\hline$a^{1} P-b^{1} D$ & $1-2$ & 27661.02 M1 & $1.79(-2)$ \\
\hline \multirow[t]{2}{*}{$a^{1} \mathrm{P}-c^{3} \mathrm{~F}$} & $1-2$ & 7581.83 M1,E2 & $4.62(-3)$ \\
\hline & & $7520.05 \mathrm{E} 2$ & $3.90(-3)$ \\
\hline$a^{1} P-a^{1} F$ & $1-3$ & $6056.86 \mathrm{E} 2$ & $5.39(-3)$ \\
\hline$a^{1} H-a^{1} F$ & $5-3$ & $7392.46 \mathrm{E} 2$ & $3.19(-2)$ \\
\hline \multirow[t]{2}{*}{$b^{1} D-c^{3} F$} & $2-2$ & 10444.72 M1 & $2.46(-3)$ \\
\hline & $2-3$ & 10327.83 M1,E2 & $5.09(-3)$ \\
\hline \multirow{3}{*}{$a^{5} D-a^{5} D$} & $4-3$ & $161611.29 \mathrm{M} 1$ & $7.67(-3)$ \\
\hline & $3-2$ & 235729.18 M1 & $4.80(-3)$ \\
\hline & $2-1$ & 369448.43 M1 & $1.85(-3)$ \\
\hline \multirow[t]{2}{*}{$c^{3} \mathrm{~F}-\mathrm{a}^{1} \mathrm{~F}$} & $2-3$ & 30113.81 M1 & $2.51(-3)$ \\
\hline & $4-3$ & 32843.85 M1 & $1.90(-3)$ \\
\hline
\end{tabular}

${ }^{a}$ Wavelengths, in air, are deduced from the observed energy levels compiled by Sugar \& Corliss (1985).

* Cancellation effects present (see text).

interference effects that cause a weak line to become still weaker or a strong line to nearly disappear. Consequently, in making numerical calculations on individual transitions, it is worthwhile evaluating the cancellation factors, CF, as defined by Cowan (1981). Small values of CF factors (typically $\mathrm{CF} \leq 0.01$ ) indicate that the corresponding lines may be expected to show large percent errors. Such transitions are indicated by starred $A$-values in Table 3 .

Although it is well established that the neglect of some configurations is partly overcome by scaling down the Slater integrals and by fitting the calculated energy levels to the observed values, the effects of configurations not included explicitly in our physical model due to computer memory limitations were estimated in separate semiempirical HFR calculations. In particular, the $3 \mathrm{~s} 3 \mathrm{p}^{6} 3 \mathrm{~d}^{9}$, $3 \mathrm{~s} 3 \mathrm{p}^{6} 3 \mathrm{~d}^{8} 4 \mathrm{~s}$ and $3 \mathrm{~s} 3 \mathrm{p}^{6} 3 \mathrm{~d}^{7} 4 \mathrm{~s}^{2}$ configurations were investigated. As mentioned recently by Quinet \& Hansen (1995), these configurations, corresponding to the core excitation $3 \mathrm{~s} \rightarrow 3 \mathrm{~d}$ in $3 \mathrm{~s}^{2} 3 \mathrm{p}^{6} 3 \mathrm{~d}^{8}, 3 \mathrm{~s}^{2} 3 \mathrm{p}^{6} 3 \mathrm{~d}^{7} 4 \mathrm{~s}$ and $3 \mathrm{~s}^{2} 3 \mathrm{p}^{6} 3 \mathrm{~d}^{6} 4 \mathrm{~s}^{2}$, might contribute to the transition probabilities for the forbidden lines considered in the present work. By comparing a three-configuration calculation (including $3 \mathrm{~d}^{8}, 3 \mathrm{~d}^{7} 4 \mathrm{~s}$ and $3 \mathrm{~d}^{6} 4 \mathrm{~s}^{2}$ ) with a six-configuration calculation (adding $3 \mathrm{~s} 3 \mathrm{p}^{6} 3 \mathrm{~d}^{9}, 3 \mathrm{~s} 3 \mathrm{p}^{6} 3 \mathrm{~d}^{8} 4 \mathrm{~s}$ and $3 \mathrm{~s} 3 \mathrm{p}^{6} 3 \mathrm{~d}^{7} 4 \mathrm{~s}^{2}$ ), the effect on the $A$-values of the latter three configurations was estimated.
In general, for the strongest multiplets $\left(A_{\text {multiplet }} \geq\right.$ $\left.0.01 \mathrm{~s}^{-1}\right)$, both calculations agree within a few percent if we except $a^{3} F-b^{1} G, a^{3} F-a^{1} P, a^{3} F-b^{1} D, b^{3} F-a^{1} P$, $a^{3} \mathrm{P}-\mathrm{c}^{3} \mathrm{P}, \quad \mathrm{a}^{5} \mathrm{P}-\mathrm{b}^{3} \mathrm{P}, \quad \mathrm{a}^{5} \mathrm{P}-\mathrm{a}^{3} \mathrm{D}, \quad \mathrm{a}^{3} \mathrm{G}-\mathrm{a}^{3} \mathrm{G}, \quad \mathrm{b}^{3} \mathrm{P}-\mathrm{a}^{1} \mathrm{P}$, $\mathrm{b}^{3} \mathrm{P}-\mathrm{b}^{1} \mathrm{D}, \mathrm{a}^{3} \mathrm{D}-\mathrm{a}^{1} \mathrm{~F}$ and $\mathrm{a}^{1} \mathrm{P}-\mathrm{b}^{1} \mathrm{D}$ for which the multiplet $A$-values were $20-30 \%$ larger when calculated with the six-configuration expansion. Exceptions occur also for the $a^{3} F-a^{5} D, a^{1} G-b^{1} D$ and $c^{3} P-c^{3} F$ lines for which the transition probabilities were reduced by about $25 \%$ when adding the $3 \mathrm{~s} 3 \mathrm{p}^{6} 3 \mathrm{~d}^{9}, 3 \mathrm{~s} 3 \mathrm{p}^{6} 3 \mathrm{~d}^{8} 4 \mathrm{~s}$ and $3 \mathrm{~s} 3 \mathrm{p}^{6} 3 \mathrm{~d}^{7} 4 \mathrm{~s}^{2}$ configurations.

Table 4. Comparison of the transition probabilities, $A_{k i}$ in $\mathrm{s}^{-1}$, calculated in the present work (HFR) with those obtained by Nussbaumer \& Storey (1988b) (NS) for the forbidden lines connecting the lowest three terms in Co II. $A(B)$ stands for A. $10^{B}$

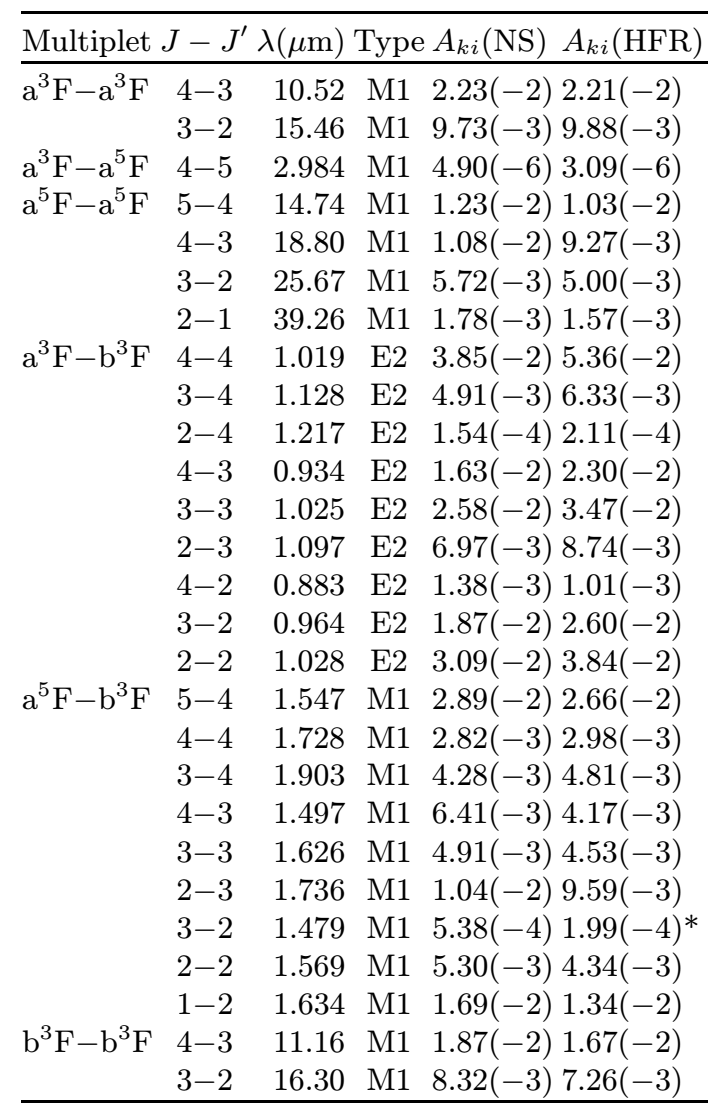

* Cancellation effects present (see text).

A very limited number of transition probabilities has been reported previously for [Co II] lines. To our knowledge, only the results obtained by Nussbaumer \& Storey (1988b) for the forbidden transitions involving the first three terms, $\mathrm{a}^{3} \mathrm{~F}, \mathrm{a}^{5} \mathrm{~F}$ and $\mathrm{b}^{3} \mathrm{~F}$, have been published. Their calculations were based on the multiconfiguration expansion of eigenfunctions as implemented in the SUPERSTRUCTURE code of Eissner et al. (1974) and 
modified by Nussbaumer \& Storey (1978). In their study, the individual configurations were constructed from oneelectron wavefunctions, the radial parts of which being calculated either in a scaled Thomas-Fermi-Dirac or a scaled Coulomb potential. The scaling parameters in the potentials were chosen to minimize the sum of the energies of the lowest three terms. Table 4 shows the comparison between the computations of Nussbaumer \& Storey (1988b) and the present work. The agreement between the two sets of results is very good (within $15 \%$ ) for the $\mathrm{a}^{3} \mathrm{~F}-\mathrm{a}^{3} \mathrm{~F}$, $a^{5} \mathrm{~F}-\mathrm{a}^{5} \mathrm{~F}, \mathrm{a}^{5} \mathrm{~F}-\mathrm{b}^{3} \mathrm{~F}$ and $\mathrm{b}^{3} \mathrm{~F}-\mathrm{b}^{3} \mathrm{~F}$ magnetic dipole transitions if we except $a^{5} F_{4}-b^{3} F_{3}$ and $a^{5} F_{3}-b^{3} F_{2}$ for which larger discrepancies are observed. The difference can even reach a factor of three for the latter transition but, in that particular case, the $A$-value computed in our work is affected by strong cancellation effects and, consequently, could be uncertain. Larger differences $(30-40 \%)$ appear also when comparing our results with those obtained by Nussbaumer \& Storey (1988b) for the $\mathrm{a}^{3} \mathrm{~F}-\mathrm{a}^{5} \mathrm{~F}$ magnetic dipole and $\mathrm{a}^{3} \mathrm{~F}-\mathrm{b}^{3} \mathrm{~F}$ electric quadrupole transitions. For these multiplets, the transitions arise through spin-orbit and configuration interactions and are therefore particularly sensitive to the choice of eigenfunctions. The magnitudes of the coefficients due to spin-orbit and CI depend on the $a^{3} F-a^{5} F$ and $a^{3} F-b^{3} F$ term separations. These term separations calculated in our work (3460 and $\left.9757 \mathrm{~cm}^{-1}\right)$ are in excellent agreement with the observations (3457 and $9774 \mathrm{~cm}^{-1}$ ) while the values obtained in intermediate coupling by Nussbaumer \& Storey (1988b), i.e. 595 and $7551 \mathrm{~cm}^{-1}$, are too small.

\section{Conclusion}

Radiative transition probabilities have been calculated for all forbidden lines connecting the 47 energetically lowest energy levels in singly ionized cobalt using a rather sophisticated atomic structure model. The new HFR data reported in this paper provide an extended and reliable physical basis for astrophysical studies where [Co II] lines play an important role. Some of our calculated $A$-values have already been included in SN Ia spectral model and have significantly improved the agreement between the synthetic spectra and supernova spectra at early times when cobalt is as abundant as iron in the supernovae (Liu et al. 1997b).

Acknowledgements. It is a pleasure to thank Dr. W. Liu and Dr. D.J. Jeffery (Oak Ridge National Laboratory) for their interest in this work and for stimulating discussions.

\section{References}

Arnett W.D., 1979, ApJ 230, L37

Arnett W.D., 1982, ApJ 253, 785

Chevalier R.A., 1981, ApJ 246, 267

Colgate S.A., McKee C., 1969, ApJ 157, 623

Colgate S.A., Petschek A.G., Kriese J.T., 1980, ApJ 237, L81

Cowan R.D., 1981, The Theory of Atomic Structure and Spectra. University of California Press, Berkeley, California

Cowan R.D., Griffin D.C., 1976, J. Opt. Soc. Am. 66, 1010

Eissner W., Jones M., Nussbaumer H., 1974, Comput. Phys. Commun. 8, 270

Fransson C., Houck J., Kozma C., 1996, Supernovae and Supernova Remnants. In: McCray R. (ed.). Cambridge University Press, Cambridge, p. 211

Garstang R.H., 1957, MNRAS 117, 393

Garstang R.H., 1958, MNRAS 118, 234

Garstang R.H., 1962, MNRAS 124, 321

Garstang R.H., 1964, J. Res. Nat. Bur. Stand., Sect. A 68, 61 Grevesse N., Nussbaumer H., Swings J.P., 1971, MNRAS 151, 239

Kirshner R.P., Oke J.B., 1975, ApJ 200, 574

Kuchner M.J., Kirshner R.P., Pinto P.A., Leibundgut B., 1994, ApJ 426, L89

Liu W., Jeffery D.J., Schultz D.R., 1997a, ApJ (in press)

Liu W., Jeffery D.J., Schultz D.R., Quinet P., Shaw J., Pindzola M.S., 1997b, ApJ (in press)

Nussbaumer H., Storey P.J., 1978, A\&A 64, 139

Nussbaumer H., Storey P.J., 1980, A\&A 89, 308

Nussbaumer H., Storey P.J., 1982, A\&A 110, 295

Nussbaumer H., Storey P.J., 1988a, A\&A 193, 327

Nussbaumer H., Storey P.J., 1988b, A\&A 200, L25

Pankey T., 1962, PhD Thesis. Howard University, Washington D.C.

Quinet P., 1996, A\&AS 116, 573

Quinet P., 1997, Phys. Scr. 55, 41

Quinet P., Le Dourneuf M., 1996, A\&AS 119, 99

Quinet P., Le Dourneuf M., Zeippen C.J., 1996a, Proceedings of the 5th International Colloquium on Atomic Spectra and Oscillator Strengths for Astrophysical and Laboratory Plasmas, Tchang-Brillet L., Wyart J.F. and Zeippen C.J. (eds.). Publications de l'Observatoire de Paris, p. 80

Quinet P., Le Dourneuf M., Zeippen C.J., 1996b, A\&AS 120, 361

Quinet P., Hansen J.E., 1995, J. Phys. B: At. Mol. Opt. Phys. 28, L213

Racah G., 1952, Phys. Rev. 85, 381

Sugar J., Corliss C., 1985, J. Phys. Chem. Ref. Data 14, Suppl. 2

Trees R.E., 1951a, Phys. Rev. 83, 756

Trees R.E., 1951b, Phys. Rev. 84, 1089

Weaver T.A., Axelrod T.S., Woosley S.E., 1980. Type I Supernovae. In: Wheeler J.C. (ed.). Austin University, Texas, p. 113

Woosley S.E., Weaver T.A., 1986, ARA\&A 24, 205 